\title{
CRECIMIENTO Y CALIDAD MORFOLÓGICA DE BERENJENA (Solanum melongena L.) EN FASE DE SEMILLERO
}

\author{
GROWTH AND MORPHOLOGICAL QUALITY OF \\ EGGPLANT (Solanum melongena L.) IN SEEDLING PHASE
}

\author{
Fernando Barraza Álvarez ${ }^{1}$ \\ Recibido para publicación: Julio 16 de 2013 - Aceptado para publicación: Noviembre 30 de 2013
}

\begin{abstract}
RESUMEN
Para conocer el efecto del remojo de semillas de berenjena en agua sobre la germinación y crecimiento de plantas en semillero, se hizo una investigación en la Universidad de Córdoba (Montería, Colombia) con la variedad criolla 'Lila', sometida a cuatro tratamientos (T): T0 (semilla sin remojo), T1 (remojo 24 horas), T2 (remojo 48 horas) y T3 (remojo 72 horas). Las semillas sin remojo presentaron porcentaje de germinación del $71 \%$, mientras que se obtuvo 85, 92 y $96 \%$ para los tratamientos T1, T2 y T3, respectivamente. A los 33 días después de la siembra, las plantas del T3 estuvieron listas para trasplante y presentaron diferencias estadísticas con respecto a los demás tratamientos, con mayores valores de longitud del tallo principal $(23 \mathrm{~cm})$, diámetro del tallo principal (3 mm), número de hojas (5), área foliar $\left(209 \mathrm{~cm}^{2}\right)$ y materia seca total $(23 \mathrm{~g})$.
\end{abstract}

Palabras clave: remojo de semillas, tratamientos pre-siembra, tasa absoluta de germinación, crecimiento vegetal.

\begin{abstract}
In order to know the effect of soaking of eggplant seeds in water on germination and plant growth in seedling, a research was made at the University of Córdoba (Monteria, Colombia) with the 'Lila' creole variety, subjected to four treatments (T): T0 (without soaking), T1 (soak for 24 hours), T2 (soak for 48 hours) and T3 (soak for 72 hours). The without soaking seeds presented germination percentage of $71 \%$, while it was obtained 85,92 and $96 \%$ for the treatments T1, T2 and T3, respectively. To the 33 days after sow, the plants of the T3 were ready to transplant and they presented statistical differences in connection with the other treatments, with highest average value in longitude of the main shoot $(23 \mathrm{~cm})$, diameter of the main shoot $(3 \mathrm{~mm})$, number of leaves (5), leaf area $\left(209 \mathrm{~cm}^{2}\right)$ and whole dry matter $(23 \mathrm{~g})$.
\end{abstract}

Key words: seed soaking, pre-plant treatments, absolute rate of germination, plant growth.

'Docente investigador, Departamento de Ingeniería Agronómica y Desarrollo Rural, Facultad de Ciencias Agrícolas. Grupo de Investigaciones en Agricultura Sostenible. Universidad de Córdoba, Carrera 6 No.76-103, Apartado Aéreo 354, Montería, Córdoba, Colombia. Autor para correspondencia: fbarra-za@correo.unicordoba.edu.co 


\section{INTRODUCCIÓN}

La mayor parte de las especies olerícolas se propagan por medio de semillas sexuales en semilleros. Allí se brindan los mejores cuidados y manejo agronómico inicial óptimo para obtener plántulas vigorosas con características fenotípicas adecuadas para el trasplante definitivo en campo y garantizar en las fases y etapas sucesivas del cultivo, el mayor rendimiento y mejor calidad en la cosecha (At George 1989 y Barraza 1998).

Se tiene conocimiento empírico valioso en las diferentes zonas agroecológicas productoras de hortalizas sobre el tiempo que las plántulas permanecen en semilleros hasta el momento del trasplante, así como de algunos indicadores establecidos por observación como número de hojas y altura de plántulas, más no hay datos precisos obtenidos de la investigación y análisis científico sobre longitud y diámetro del tallo principal, área foliar, acumulación de materia seca total y días después de la siembra transcurridos para efectuar el trasplante (Boutherin y Bron 1994; Barraza 2011).

Para la zona del Valle del Sinú, en donde se siembran hortalizas como tomate (Lycopersicon esculentum Mill.), ají dulce (Capsicum annum L.) y berenjena (Solanum melongena L.), se conoce de manera práctica que el momento de trasplante ocurre a los 20, 35 y 45 días después de la siembra en semillero, respectivamente, pero que debido a apreciaciones personales de los agricultores, propias de la percepción sensorial o factores diversos que van desde la falta de conocimiento hasta los comportamientos culturales, tienen variaciones y se aplican diferentes criterios que no muestran decisiones científicamente tomadas en lo que al manejo agronómico se refiere (Barraza 2011).

En el caso del cultivo de berenjena, el manejo agronómico comienza para la mayoría de agricultores con la siembra de semillas seleccionadas de cultivos establecidos con variedades criollas, conservación de dichas semillas en almacenamiento abierto y siembra al voleo en semilleros, con semillas sometidas en algunos casos a tratamientos para mejorar los procesos de germinación y emergencia como el remojo en agua (Barraza 2011), ya que como semillas ortodoxas, las mismas pueden presentar diferencias en germinación, lo que dificulta su uso para cultivo o mejoramiento de genotipos (Gisbert et al. 2011), con la desventaja de que además se obtiene cierta desuniformidad del material vegetal para trasplante, procedente de semillas sembradas en un mismo momento.

Como consecuencia de lo anterior, es posible también que las condiciones de almacenamiento abierto referidas para las semillas propicien durante dicho periodo mecanismos de deterioro morfológico y fisiológico que disminuyen la germinación, velocidad de crecimiento tolerancia de las plántulas a condiciones adversas. Con ello, en la fase sucesiva semillero se pueden encontrar crecimiento anormal y daños en estructuras principales de las plántulas (Delouche 2002; Pérez et al. 2008 a).

Además del remojo en agua se han realizado investigaciones para facilitar la germinación de semillas de diversas plantas hortícolas, utilizando métodos Ilamados iniciadores, de 
arranque (seedpriming) o acondicionamiento osmótico, que son tratamientos pre-siembra de remojo que permiten que las semillas mejoren su porcentaje y tasa de germinación y la emergencia de plántulas, aun cuando sean sometidas a bajos potenciales de humedad externa que limiten su hidratación y con ello afecten los procesos fisiológicos del metabolismo, resultando insuficientes para que ocurra la protrusión de la radícula a través de las cubiertas de la semilla (Nascimento 2003; Hartmann y Kester 2010).

En ese sentido se han utilizado varias sustancias para el acondicionamiento osmótico de semillas de varias plantas hortícolas, entre las que se encuentran $\mathrm{NaCl}$ (Akers et al. 1985), $\mathrm{KH}_{2} \mathrm{PO}_{4}+$ $\mathrm{KNO}_{3}$ (Nerson y Govers 1986), $\mathrm{KNO}_{3}$ (Bradford et al. 1988; Thuo 2003, Ngaroga 2003), manitol (Passam et al. 1989) y polietilenglicol entre otros (Yeoung et al. 1996). Para el caso de berenjena se han utilizado tratamientos de remojo en polietilenglicol, ácido giberélico, cytozime, $\mathrm{KNO}_{3} \mathrm{CaCl}_{2^{\prime}} \mathrm{KH}_{2} \mathrm{PO}_{4^{\prime}} \mathrm{Na}_{2} \mathrm{HPO}_{4^{\prime}}$ orina de vaca (Kumar 2005) y licor nacional de la India (Sharma y Sharma 2010; Dwivedi et al. 2012). Otros tratamientos probados simultáneamente en tomate, berenjena y ají, incluyen, además del uso de agua, otras sustancias como extracto de fríjol caupí (Vignaunguiculata (L.) Walp.), extracto de grama de caballo (Vignabiflorus Lam. Verdc.), extracto de harina de huesos, gelatina, $\mathrm{KH}_{2} \mathrm{PO}_{4^{\prime}}$ $\mathrm{KNO}_{3}, \mathrm{ZnSO}_{4}, \mathrm{FeSO}_{4}, \mathrm{Na}_{2} \mathrm{SO} 4, \mathrm{MnSO}_{4}$ y ácido indol butírico (Ponnuswamy y Vijayalakshmi 2011).

La presente investigación se hizo con el objetivo de establecer qué ventajas tiene el remojo de semillas de berenjena en agua, y conocer el tiempo de remojo más favorable para aumentar el porcentaje de germinación y cómo se comporta el crecimiento de plántulas en la fase de semillero, así como los días requeridos para el trasplante a sitio definitivo.

\section{MATERIALES Y MÉTODOS}

La investigación se realizó entre el 22 de febrero y el 26 de marzo de 2012, en el Campo Experimental de Cultivos de Hortalizas y Laboratorio de Fisiología Vegetal de la Universidad de Córdoba, ubicada en Montería, Córdoba, Colombia, a $8^{\circ} 49^{\prime} \mathrm{N}, 75^{\circ} 51^{\prime} \mathrm{W}$, altitud de $25 \mathrm{msnm}$, temperatura ambiente promedio de $28{ }^{\circ} \mathrm{C}$, precipitación promedia anual de $1.249 \mathrm{~mm}$ y humedad relativa promedio de 78\% (Weatherbase, 2013). Se utilizaron semillas de berenjena de la variedad criolla 'Lila', procedentes de cultivos sembrados en la Universidad de Córdoba (Montería, Colombia), a las cuales se aplicaron cuatro tratamientos de remojo en agua, con cambio de agua cada 24 horas: T0 (testigo absoluto sin remojo), T1 (remojo durante 24 horas), T2 (remojo durante 48 horas y T3 (remojo durante 72 horas).

\section{Bioensayo de germinación}

Para establecer el porcentaje de germinación de las semillas, se establecieron los tratamientos en diseño completamente al azar con cuatro repeticiones de 25 semillas cada una, a temperatura ambiente en el Laboratorio de Fisiología Vegetal de la Universidad de Córdoba, distribuidas en patrón circular en cajas de Petri de vidrio de $90 \mathrm{~mm}$ de diámetro, con sustrato de papel filtro humedecido con $15 \mathrm{~mL}$ de agua destilada estéril (Carrillo et al. 2009 e ISTA 2011). Se tomó como medida de germinación la emergencia de la radícula, de acuerdo con 
lo indicado por Torres et al. (2008). Se hicieron conteos diarios de semillas germinadas, a partir del momento de la colocación en cajas de Petri hasta los 15 días posteriores, y se estableció el porcentaje de germinación por día (Nieto 2005), con modelación mediante el modelo logístico:

$$
y=\frac{A}{\left(1+B \cdot e^{-C \cdot x}\right)}
$$

donde y: variable respuesta; A: máximo valor observado; B: no tiene significado biológico, y solo toma lugar en el tiempo inicial, cuando $\mathrm{x}=$ $0 ; x=$ días después del trasplante; C: parámetro relacionado con el valor de $\mathrm{x}$ para el punto de inflexión; e: constante matemática, base del logaritmo natural $=2.718281828$. La tasa absoluta de germinación se calculó a través de la primera derivada del modelo logístico (Calvo et al. 1994).

\section{Bioensayo de análisis de crecimiento de plántulas}

Para cada uno de los tratamientos se sembraron 700 semillas por repetición en semilleros de $0,90 \mathrm{~cm}$ de ancho por 1,0 $\mathrm{m}$ de longitud, distribuidos en un diseño completamente al azar con cuatro repeticiones por tratamiento. La separación entre semilleros fue de 1,0 m. El área total del experimento fue de 46,2 $\mathrm{m}^{2}$. Las semillas se sembraron en hileras transversales (surcos), separadas a $1 \mathrm{~cm}$ entre sí, con distancia entre surcos de $10 \mathrm{~cm}$, a una profundidad de $0,5 \mathrm{~cm}$. El sustrato utilizado fue una mezcla en proporción volumétrica 1:1:1 (tierra de aluvión, arena de río, cascarilla de arroz). A partir de los 12 días posteriores a la siembra, se tomaron las siguientes variables, cada 3 días: altura de planta, a dos plántulas (Carrillo et al. 2009 e ISTA 2011) marcadas en los tres surcos centrales de cada repetición (se midió en $\mathrm{cm}$ la longitud desde el cuello de la raíz hasta el ápice de la hoja más larga), diámetro del tallo principal (a las mismas dos plántulas anteriores en cada repetición se les midió en $\mathrm{mm}$ el grosor del tallo principal a 1 cm de la base de la plántula), número de hojas (se hizo conteo del número de hojas verdaderas completamente expandidas a las mismas dos plántulas anteriores en cada repetición), área foliar $\left(\mathrm{AF}\right.$, en $\left.\mathrm{cm}^{2}\right)$, a través de procesamiento digital con el software DDA (Ferreira et al. 2008), acoplado a scanner HP Scanjet $₫ 3400$ C, a tres plántulas seleccionadas de los tres surcos centrales de cada repetición), materia seca total (MS) y materia seca de hojas a las mismas plántulas anteriores mediante secado en horno eléctrico a $85^{\circ} \mathrm{C}$ hasta obtención de peso constante.

El análisis estadístico se llevó a cabo con análisis de varianza y pruebas de comparación de medias de Tukey $(\mathrm{P} \leq 0.05)$ mediante el software SAS versión 10.0 (SAS Institute 2008).Por las características de la información obtenida en las variables del bioensayo, para generar los modelos y gráficas del crecimiento, se aplicó el procedimiento de regresión no lineal.

\section{RESULTADOS Y DISCUSIÓN}

\section{Porcentaje y tasa absoluta de germinación}

El porcentaje y la tasa absoluta de germinación aumentaron a través del tiempo transcurrido después de la siembra y presentaron valores más altos conforme aumentó el periodo de remojo de las semillas. Para el caso del T3 (remojo 72 horas), la germinación comenzó un día antes que el resto de tratamientos y finalizó 
12 días después de la siembra, mientras que para los demás tratamientos finalizó a los 14 días después de la siembra (Tabla 1).

La trayectoria gráfica de la germinación a través del tiempo fue sigmoide y la tasa absoluta de germinación siguió una distribución normal (Figura 1), propia de dicho proceso, en donde se

Tabla 1. Comparación de medias del porcentaje de germinación de semillas de berenjena variedad criolla 'Lila' sometidas a cuatro tratamientos con remojo en agua.

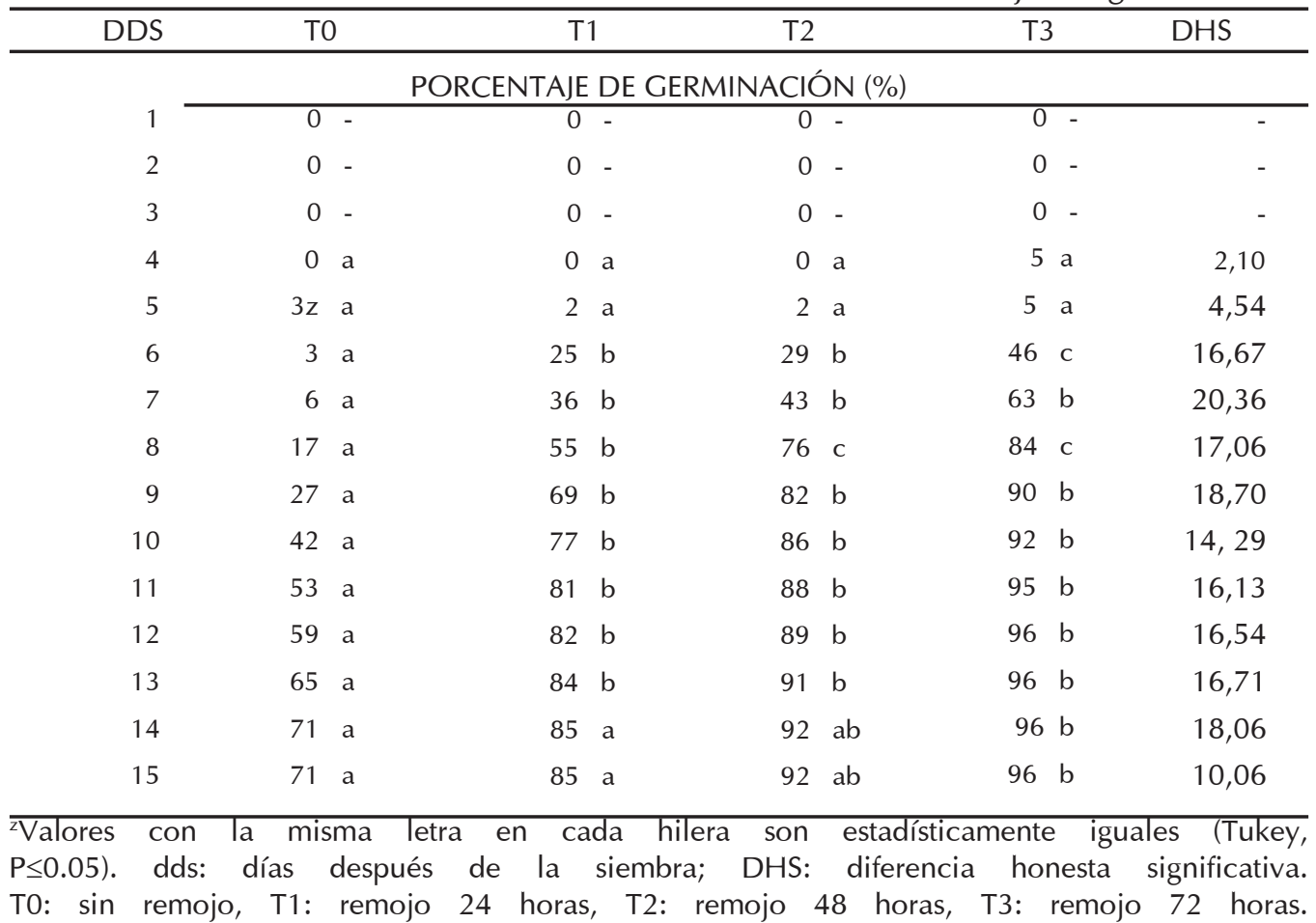

observó que para todos los tratamientos aumentó progresivamente a través del tiempo, alcanzó un valor máximo y de allí comenzó a descender progresivamente hasta alcanzar sus valores mínimos (Hunt 1982), lo que coincide con resultados en Solanum melongena L. cv.híbrido Arka Navneet (Kumar 2005), Cucumis sativus L. (Gómez et al. 2003), Physalisixocarpa Brot. (Pérez-Camacho et al. 2008 a; Pérez-Camacho et al. 2008 b; Pichardo et al. 2010) y Cleomegynandra L. (Kamotho et al. 2003).

A los 6 días después de la siembra, el tratamiento de remojo de semillas durante 72 horas (T3) superó con diferencias estadísticas altamente significativas al resto de tratamientos. Durante las demás fechas, los tratamientos T2 (remojo 48 horas) y T3 (remojo 72 horas) fueron estadísticamente iguales entre sí y superiores en grado altamente significativo a los tratamientos T0 (sin remojo) y T1 (remojo 24 horas) (Tabla 1). De acuerdo con los datos de la Tabla 1 y las trayectorias gráficas de la Figura 1, se observó que el tratamiento 3 (remojo 72 horas) presentó los valores promedios más altos en porcentaje y tasa absoluta de germinación, de tal manera que con dicho tratamiento a los 6 dds se presentó la mayor tasa absoluta de germinación, y ya habían germinado casi la mitad de las semillas sembradas y con mayor 
velocidad durante el periodo de germinación, que representa importancia desde los puntos de vista agronómico y fisiológico en la medida que se presentó un incremento rápido y uniforme en la germinación y emergencia, lo que favorece a futuro el establecimiento en sitio definitivo (Thuo 2003), con la ventaja de mayor crecimiento y extensión radicular, lo que puede llegar a aumentar el rendimiento (Kumar 2005).
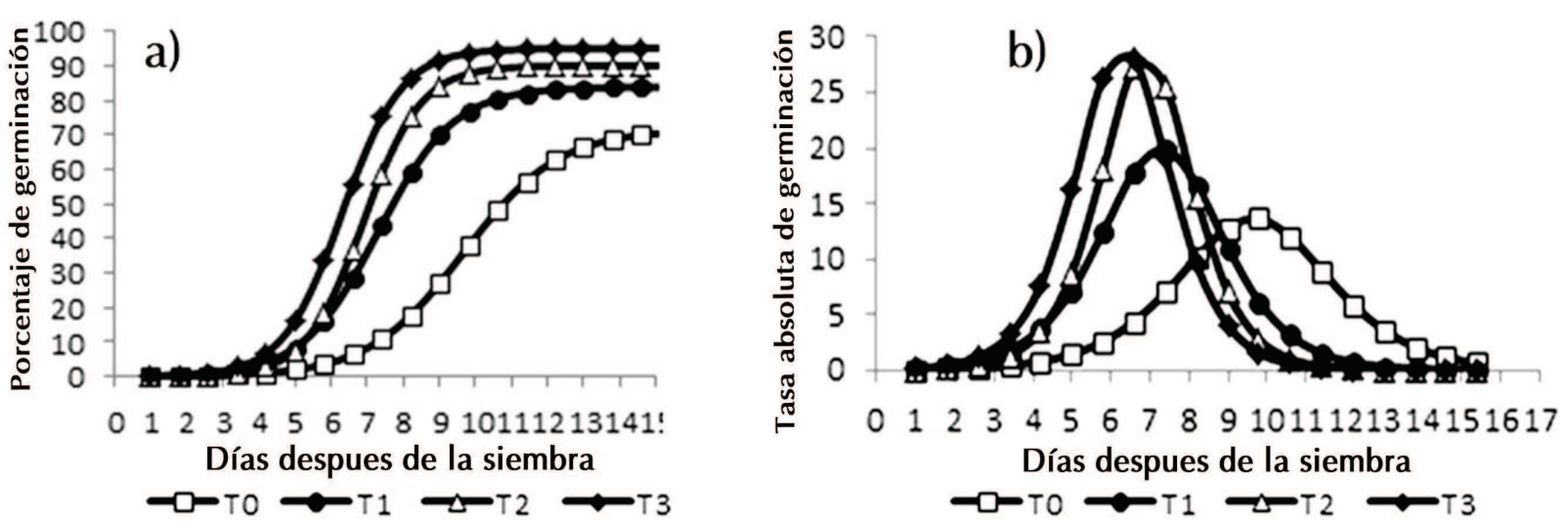

Figura 1. Porcentaje de germinación (a) y tasa absoluta de germinación (b) de semillas de berenjena en cuatro tratamientos de remojo en agua.

Además de lo anterior, el remojo de semillas de berenjena con agua, contribuyó a mejorar la germinación, que como especie de comportamiento ortodoxo en este sentido (Gisbert et al. 2011) presentó en condiciones normales el $71 \%$ (T0), y que incrementó en 14,21 y $25 \%$ con el remojo durante 24,48 y 72 horas, respectivamente. Los tratamientos con remojo en agua durante 48 y 72 horas, superaron la mejora de la germinación de semillas que se reporta con el remojo en licor nacional de la India, que es del $20 \%$, y que se indica como un método tradicional económico y efectivo (Sharma y Sharma 2010).

\section{Variables morfológicas del crecimiento y acumulación de materia seca}

El crecimiento de las plántulas de berenjena estudiado a través de las variables longitud y diámetro del tallo principal, número de hojas, área foliar y acumulación de materia seca de hojas y total, presentó una tendencia general al aumento a medida que transcurrió el tiempo después de la siembra de las semillas. La trayectoria seguida para todas estas variables de acuerdo con las gráficas correspondió a un comportamiento sigmoidal (Figura 2), propio del aumento en tamaño que implica el proceso de crecimiento a través del tiempo y que se debe al mayor número de células generadas a través del proceso de mitosis, seguida de la expansión de las mismas una vez que se han iniciado los procesos fisiológicos a nivel bioquímico que se estimulan con el inicio de la germinación de las semillas (Nascimento 2003).

El proceso de crecimiento de plántulas posterior a la germinación de las semillas sometidas a remojo se vio favorecido por dicha práctica, 

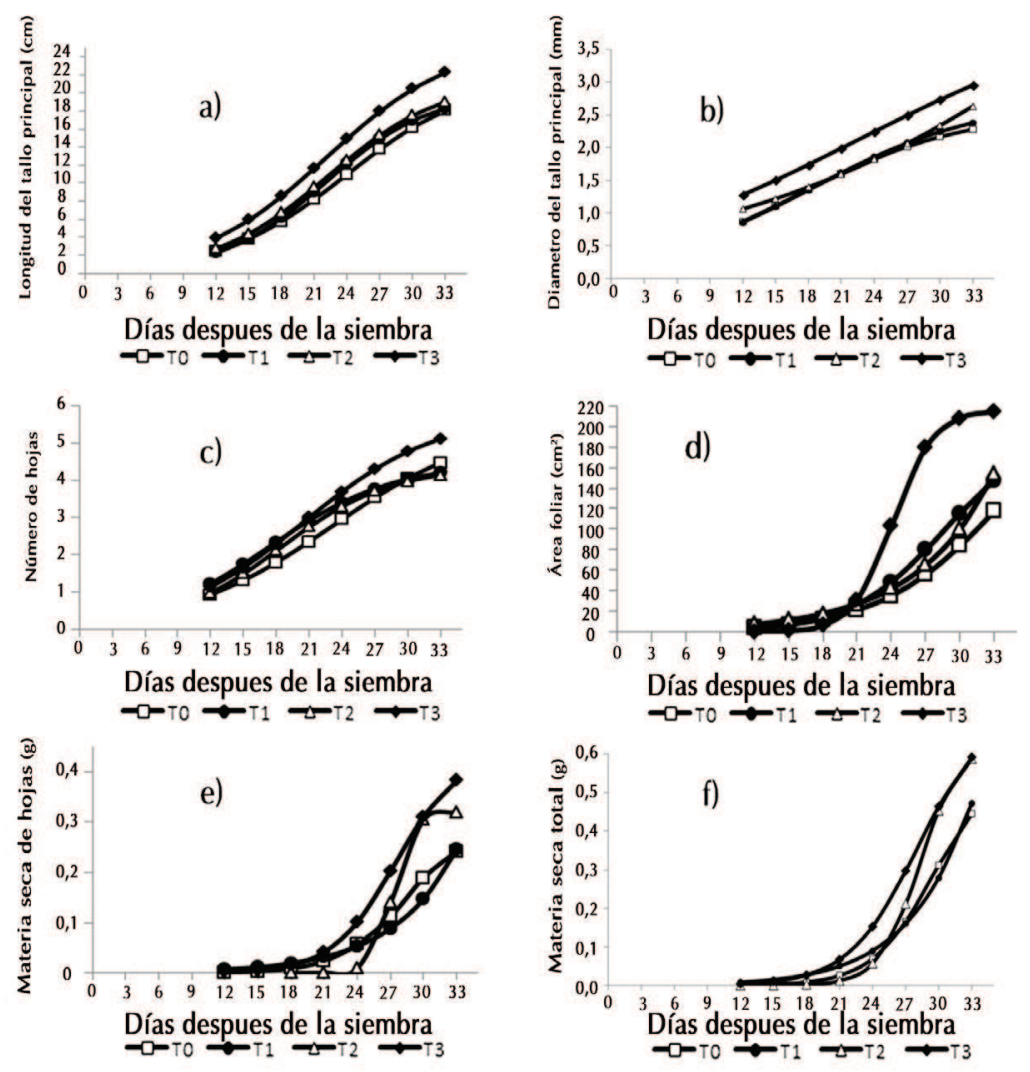

Figura 2. Crecimiento de plántulas de berenjena procedentes de semillas sometidas a cuatro tratamientos de remojo en agua: a) longitud del tallo principal, b) diámetro del tallo principal, c) número de hojas, d) área foliar, e) materia seca de hojas y f) materia seca total.

ya que durante la germinación que se da en condiciones normales, las semillas primero deben humedecerse a través del proceso de imbibición del agua que se encuentre disponible en el sustrato, proceso que queda limitado hasta el momento que el contenido de humedad no esté disponible (Hartmann y Kester 2010), mientras que con el remojo de semillas en agua, hay permanente e ininterrumpida actividad bioquímica en la semilla, propia del proceso de germinación que ocurre de manera irreversible hasta terminar con la emergencia de la plántula (White Harvest Seed Company 2013).
Todas las variables morfológicas indicadas, así como la acumulación de materia seca de hojas y total se ajustaron al modelo logístico (Tabla 2), con coeficientes de correlación bastante cercanos a la unidad, lo que refleja la bondad de ajuste de los modelos para conocer el patrón de comportamiento en el tiempo respecto a las variables representadas (Brand y Weetman 1987). En estudios con otras hortalizas como tomate de cáscara (Cuenca et al. 1999), chile poblano (Martínez 2006), lechuga (Carranza et al. 2009) y pepino (Barraza 2012), se han obtenido resultados similares de ajuste de variables morfológicas del crecimiento con el modelo logístico. 
Tabla 2. Modelos logísticos para estimar crecimiento de plántulas de berenjena (Solanum melongena L.) procedentes de semillas sometidas a cuatro tratamientos con remojo en agua.

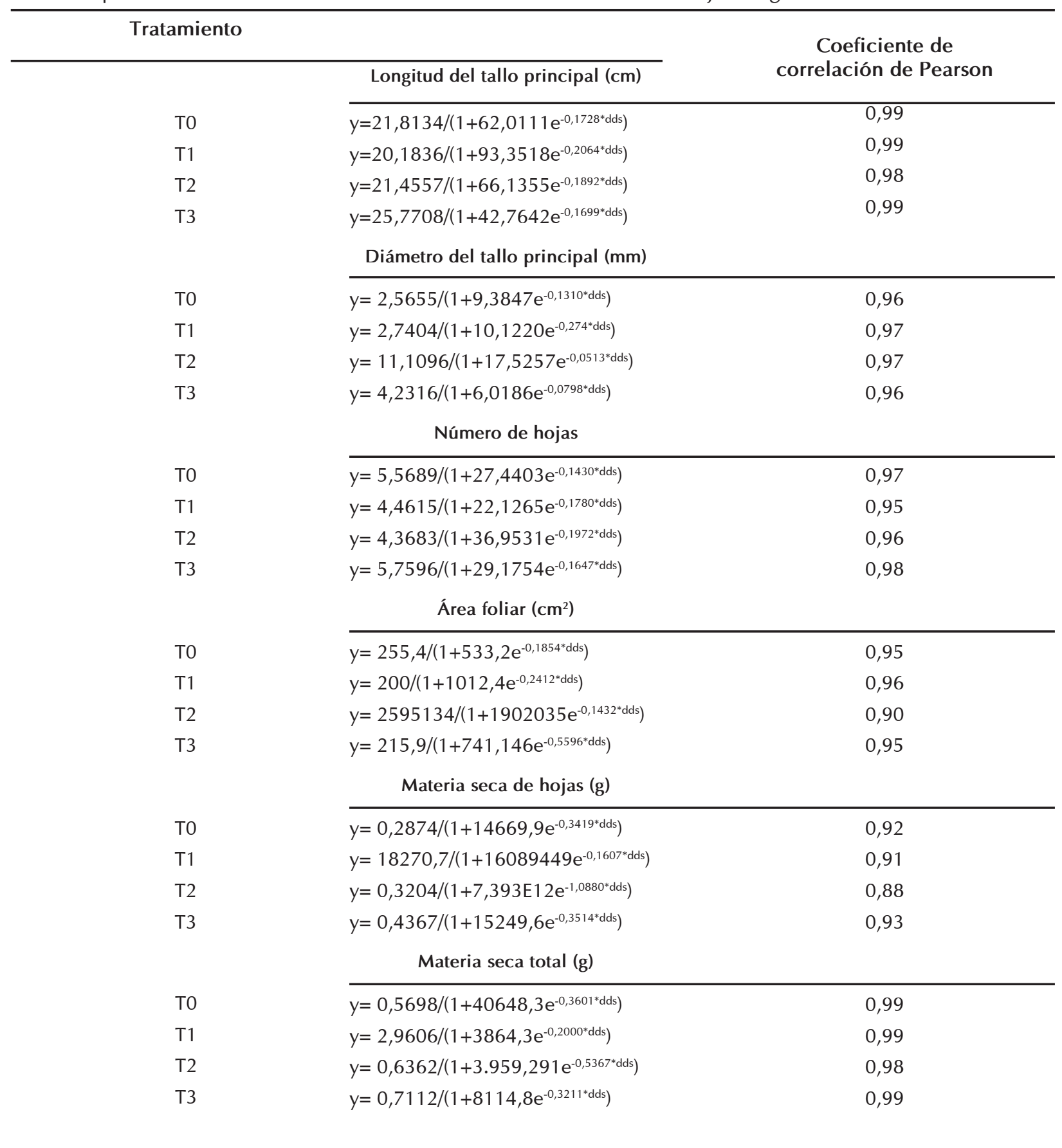

y: variable del crecimiento (longitud del tallo principal, diámetro del tallo principal, número de hojas, área foliar, acumulación de materia seca de hojas y total); dds: días después de la siembra. T0: sin remojo, T1: remojo 24 horas, T2: remojo 48 horas, T3: remojo 72 horas. 
De acuerdo con la Tabla 3, se presentaron diferencias estadísticas significativas entre tratamientos en lo que respecta a longitud y diámetro del tallo principal, número de hojas y área foliar. Para longitud del tallo principal el tratamiento con remojo de semillas durante 72 horas (T3) superó significativamente $(\mathrm{P} \leq 0.05)$ al resto de tratamientos a los 12,18 dds y en el periodo comprendido entre los 21 y 33 dds. Para el caso de diámetro del tallo principal, T3 superó con diferencias estadísticas $(\mathrm{P} \leq 0.05)$ a los demás tratamientos entre los 15 y 33 dds.

De igual forma, T3 fue superior en grado significativo $(P \leq 0.05)$ al resto de tratamientos en lo que respecta a número de hojas (30 y 33 dds) y área foliar (21 a 33 dds).

De acuerdo con lo anterior, el mayor crecimiento presentado a través de las variables morfológicas de las plántulas obtenidas con remojo de semillas durante 72 horas (T3), pudo estar relacionado con la mejora en la calidad que transmiten a las semillas los tratamientos de presiembra, ya que en este orden de ideas, en concordancia con Kumar (2005), el remojo de semillas en agua y otras sustancias, aumenta significativamente el porcentaje de germinación, velocidad de germinación, longitud y ramificación de las raíces, índice de vigor, materia seca y porcentaje de emergencia, lo cual a nivel fisiológico es debido al alargamiento de embriones, alta tasa de actividad enzimática, metabólica (en lo que concierne principalmente a translocación de azúcares, hidrólisis de proteínas y oxidación, entre otros procesos) y respiración, así como la mejor utilización y movilización de metabolitos

Tabla 3. Comparación de medias de longitud y diámetro del tallo principal, número de hojas y área foliar de plántulas de berenjena en fase de semillero, procedentes de semillas sometidas a cuatro tratamientos con remojo en agua

\begin{tabular}{|c|c|c|c|c|c|c|c|c|c|}
\hline $\mathrm{DDS}$ & $\mathrm{T}$ & & $\overline{\mathrm{T}}$ & & $\overline{\mathrm{T}}$ & & & & $\overline{\mathrm{DHS}}$ \\
\hline \multicolumn{10}{|c|}{ Longitud del tallo principal $(\mathrm{cm})$} \\
\hline 12 & $2,90^{2}$ & $\mathrm{a}$ & 2,75 & $\mathrm{a}$ & 3,53 & $a b$ & 3,98 & $\mathrm{ab}$ & $\overline{0,99}$ \\
\hline 15 & 4,15 & a & 3,98 & a & 4,58 & a & 5,61 & a & 2,40 \\
\hline 18 & 5,55 & a & 5,93 & a & 6,08 & $\mathrm{a}$ & 7,93 & a & 1,80 \\
\hline 21 & 7,35 & a & 8,13 & a & 8,65 & $a b$ & 12,45 & $a b$ & 0,94 \\
\hline 24 & 11,75 & a & 13,58 & b & 13,85 & $\mathrm{~b}$ & 16,20 & $\mathrm{~b}$ & 1,44 \\
\hline 27 & 13,48 & $\begin{array}{l}\mathrm{a} \\
\mathrm{a}\end{array}$ & 14,20 & a & $\begin{array}{l}3,05 \\
15,00\end{array}$ & $a b$ & 16,80 & $\mathrm{ab}$ & 1,25 \\
\hline 30 & 16,75 & $\mathrm{a}$ & 17,18 & a & 17,75 & $a b$ & 19,43 & $a b$ & 0,93 \\
\hline 33 & 17,70 & a & 18,20 & a & 18,75 & a & 23,23 & a & 1,30 \\
\hline \multicolumn{10}{|c|}{ Diámetro del tallo principal (mm) } \\
\hline $12^{-}$ & 0,88 & $\bar{a}$ & 0,98 & $\mathrm{a}$ & 1,13 & $a$ & 1,13 & $\mathrm{a}$ & $\overline{0,45}$ \\
\hline 15 & 1,21 & a & 1,08 & a & $\begin{array}{l}1,25 \\
1,25\end{array}$ & $a b$ & 1,48 & b & 0,31 \\
\hline 18 & 1,28 & a & 1,25 & a & 1,30 & a & 1,93 & b & 0,46 \\
\hline 21 & 1,40 & a & 1,44 & a & 1,40 & a & 2,20 & b & 0,20 \\
\hline 24 & 2,03 & a & 2,05 & a & 2,03 & a & 2,20 & b & 0,10 \\
\hline 27 & 2,05 & a & 2,15 & $\begin{array}{l}\mathrm{a} \\
\mathrm{a}\end{array}$ & 2,15 & a & 2,30 & b & 0,14 \\
\hline 30 & $\begin{array}{l}2,18 \\
2,18\end{array}$ & $\begin{array}{l}\mathrm{d} \\
\mathrm{a}\end{array}$ & 2,25 & ab & $\begin{array}{l}2,13 \\
2,23\end{array}$ & $\begin{array}{l}\mathrm{d} \\
\mathrm{a}\end{array}$ & 2,55 & $\mathrm{ab}$ & 0,35 \\
\hline 33 & 2,23 & a & 2,30 & a & 2,65 & $\mathrm{a}$ & 3,15 & b & 0,44 \\
\hline \multicolumn{10}{|c|}{ Número de hojas } \\
\hline 12 & 1,25 & $a$ & 1,50 & $\mathrm{a}$ & 1,25 & $a$ & 1,25 & $\mathrm{a}$ & 1,09 \\
\hline 15 & 1,25 & $\begin{array}{l}\mathrm{d} \\
\mathrm{a}\end{array}$ & 1,75 & $\begin{array}{l}\mathrm{a} \\
\mathrm{a}\end{array}$ & $\begin{array}{l}1,25 \\
1,25\end{array}$ & $\begin{array}{l}\mathrm{a} \\
\mathrm{a}\end{array}$ & 1,75 & $\begin{array}{l}\mathrm{a} \\
\mathrm{a}\end{array}$ & 1,05 \\
\hline 18 & 1,75 & a & 2,00 & a & 2,00 & a & 2,00 & a & 0,53 \\
\hline 21 & 2,00 & a & 2,75 & b & 3,00 & b & 3,00 & b & 0,53 \\
\hline 24 & 3,00 & a & 3,75 & b & 3,00 & $\mathrm{a}$ & 4,00 & b & 0,53 \\
\hline 27 & 3,75 & a & 4,00 & a & 4,00 & a & 4,00 & a & 0,53 \\
\hline 30 & 4,25 & $\begin{array}{l}\mathrm{a} \\
\mathrm{a}\end{array}$ & 4,00 & $\begin{array}{l}\mathrm{a} \\
\mathrm{a}\end{array}$ & 4,00 & a & 5,00 & b & 0,53 \\
\hline 33 & 4,25 & a & 4,00 & a & 4,00 & a & 5,00 & b & 0,53 \\
\hline \multicolumn{10}{|c|}{ Área foliar $\left(\mathrm{cm}^{2}\right)$} \\
\hline $12^{-}$ & 0,19 & $\bar{a}$ & 0,61 & $\mathrm{a}$ & 1,11 & $\mathrm{a}$ & 1,17 & $\bar{a}$ & 1,57 \\
\hline 15 & 3,19 & a & 3,74 & a & 3,35 & a & 5,06 & a & 3,94 \\
\hline 18 & 11,80 & $\begin{array}{l}\mathrm{d} \\
\mathrm{a}\end{array}$ & 15,53 & $\begin{array}{l}\mathrm{d} \\
\mathrm{a}\end{array}$ & 13,61 & a & 18,15 & a & 8,04 \\
\hline 21 & 18,67 & $\mathrm{a}$ & 30,67 & $\mathrm{a}$ & 29,80 & b & 43,65 & $\mathrm{c}$ & 7,07 \\
\hline 24 & 42,23 & a & 53,82 & a & 46,90 & a & 85,80 & b & 15,87 \\
\hline 27 & 57,61 & a & 66,31 & a & 7909 & $a b$ & 198,61 & c & 15,85 \\
\hline 30 & 77,65 & a & 126,27 & b & $76^{\prime} 98$ & $\mathrm{a}$ & 203,58 & c & 18,55 \\
\hline 33 & 19,73 & $\begin{array}{l}\mathrm{a} \\
\mathrm{a}\end{array}$ & 144,50 & b & $\begin{array}{l}183,29 \\
183,29\end{array}$ & $\mathrm{c}$ & 208,91 & d & 13,22 \\
\hline
\end{tabular}

${ }^{2}$ Valores con la misma letra en cada hilera son estadísticamente iguales (Tukey, P 0.05). dds: días después de la siembra; DHS: diferencia honesta significativa. 
hacia los puntos de crecimiento.

Particularmente se resaltan las amplias diferencias de área foliar encontradas entre tratamientos (Tabla 3 y Figura 2), en donde T3 superó a los demás a partir de los 21 dds y hasta el momento de trasplante (33 dds), lo que está relacionado a la vez con la mayor acumulación de materia seca total que se presentó con T3, la cual superó con diferencias estadísticas significativas a los demás tratamientos en el periodo comprendido entre 18 a 33 dds (Tabla 4 ), en vista de que una gran parte del aumento en peso seco se traduce estructuralmente en un aumento foliar que conduce a mayor crecimiento (Rojas y Rovalo 1985; Roberts et al. 1985), debido a que la ganancia de las hojas en materiales almacenados hace presión sobre la fuente fisiológica de la planta, y que además la consecuente formación de buenos tallos y hojas también conduce a más fotosíntesis, ya que como indica Bidwell (1993), los meristemos apicales producen un mayor número de hojas, ramas y otros apéndices del tallo que durante los estados juveniles de la planta ayudan a producir e invertir gran cantidad de asimilados en las hojas para mantener el crecimiento posterior (Hsiao 1982; Kiran et al. 2010).

De manera global se puede indicar que el mayor crecimiento en variables morfológicas y la mayor acumulación de materia seca de plántulas de berenjena, que aumentó a medida que lo hizo el tiempo de remojo, para dar el mejor resultado con el mayor tiempo (T3, 72 horas), se debió a la mejora propiciada por el tratamiento de presiembra en la intensificación de procesos hidrolíticos, mayor toma de nutrimentos propiciados y estimulados por la hidratación durante el establecimiento de las semillas y que en consecuencia la mayor acumulación de materia seca total es un indicativo del aumento en el vigor de las semillas (Bassi et al. 2007).

Tabla 4. Comparación de medias de acumulación de materiạ seca de hojas y materia seca total de plántulạs de berenjena en fase de semillero, procedentes de semillas sometidas a cuatro tratamientos con remojo en agua.

\begin{tabular}{|c|c|c|c|c|c|c|c|c|c|}
\hline DDS & \multicolumn{2}{|c|}{ T0 } & \multicolumn{2}{|c|}{ T1 } & \multicolumn{2}{|c|}{$\mathrm{T2}$} & \multicolumn{2}{|c|}{ T3 } & DMS \\
\hline \multicolumn{10}{|c|}{ Materia seca de hojas $(\mathrm{g})$} \\
\hline 12 & $0,0014^{z}$ & a & 0,0013 & $\bar{a}$ & 0,0017 & $\mathrm{a}$ & 0,0028 & $\mathrm{a}$ & 0,0015 \\
\hline 15 & 0,0038 & a & 0,0038 & a & 0,0056 & a & 0,0158 & $\mathrm{a}$ & 0,0042 \\
\hline 18 & 0,0157 & a & 0,0170 & a & 0,0171 & b & 0,0292 & b & 0,0081 \\
\hline 21 & 0,0279 & a & 0,0408 & a & 0,0471 & b & 0,0627 & b & 0,0165 \\
\hline 24 & 0,0668 & a & 0,0623 & a & 0,0566 & b & 0,0988 & b & 0,0300 \\
\hline 27 & 0,0933 & a & 0,0885 & a & 0,1153 & b & 0,1646 & b & 0,0569 \\
\hline 30 & 0,2119 & a & 0,1363 & b & 0,3420 & $\mathrm{c}$ & 0,3583 & c & 0,0729 \\
\hline 33 & 0,2341 & a & 0,2491 & $\mathrm{a}$ & 0,2880 & $a b$ & 0,3623 & $a b$ & 0,0791 \\
\hline \multicolumn{10}{|c|}{ Materia seca total (g) } \\
\hline 12 & 2,9000 & a & 2,7500 & $\mathrm{a}$ & 3,5250 & a & 3,9750 & $\mathrm{a}$ & 0,9896 \\
\hline 15 & 4,1500 & a & 3,9750 & a & 4,5750 & a & 5,6125 & a & 2,3972 \\
\hline 18 & 5,5500 & a & 5,9250 & a & 6,0750 & a & 7,9250 & b & 1,7955 \\
\hline 21 & 7,3500 & a & 8,1250 & a & 8,6500 & $\mathrm{a}$ & 12,4500 & b & 0,9426 \\
\hline 24 & 11,7500 & $\mathrm{a}$ & 13,5750 & b & 13,8500 & b & 16,2000 & C & 1,4406 \\
\hline 27 & 13,4750 & a & 14,2000 & a & 15,0000 & $\mathrm{a}$ & 16,8000 & $b$ & 1,2494 \\
\hline 30 & 16,7500 & $\mathrm{a}$ & 17,1750 & a & 17,7500 & a & 19,4250 & $b$ & 0,9263 \\
\hline 33 & 17,7000 & $\mathrm{a}$ & 18,2000 & a & 18,7500 & a & 23,2250 & b & 1,3027 \\
\hline
\end{tabular}

la siembra; DHS: diferencia honesta significativa. 
CONCLUSIONES

El remojo de semillas de berenjena en agua durante más tiempo mostró beneficios en cuanto al aumento del porcentaje y tasa absoluta de germinación, como también influyó de manera favorable en las plántulas en fase de semillero, de tal forma que con 72 horas de remojo, se obtuvo material para trasplante a los 33 dds, con mayores valores de las variables de crecimiento y acumulación de materia seca.

\section{REFERENCIAS}

Akers, S., Brede, J. and Bates, J. 1985. Why some vegetable seed cannot be primed in aerated solutions. HortScience 20: 549.

At, R. 1989. Producción de Semillas de plantas hortícolas. Ediciones Mundi-Prensa. Madrid, España. 330 p.

Barraza, F. 1998. Manejo y desinfección de semilleros y sustratos para la siembra de especies hortícolas. Universidad de Córdoba. Facultad de Ciencias Agrícolas. Departamento de Publicaciones Universidad de Córdoba. Montería, Colombia. $56 \mathrm{p}$.

Barraza, F. 2011. Diagnóstico preliminar sobre aspectos agronómicos, sociales y culturales en la siembra de hortalizas en el Valle del Sinú medio. Universidad de Córdoba. Facultad de Ciencias agrícolas. $105 \mathrm{p}$.

Barraza, F. 2012. Acumulación de materia seca del cultivo de pepino (Cucumis sativus L.) en invernadero. TemasAgrarios
17 (2): 18-29.

Bassi, G., Kanwar, J. and Singh, P. 2007. Seed enhancement treatments and seed quality in brinjal (Solanummelongena). Veg. Sci. 34 (2): 202-203.

Bidwell, R. 1993. Fisiología Vegetal. A.G.T. Editor S.A., México, D.F. 784 p.

Boutherin, D. and Bron, G. 1994. Multiplicación de plantas hortícolas. Editorial Acribia, S.A. Zaragoza, España. 223 p.

Bradford, K., May, D., Hoyle, B., Skibinski, Z., Scott, S. and Tyler, K. 1988. Seed and soil treatments to improve emergence of muskmelon from cold or crusted soils. CropScience 28 (6): 1001-1005.

Brand, G. and Weetman, F. 1987. Growth analysis of perennial plants: the relative pro-duction and its yield components. Annals of Botany 59: 45-53.

Calvo, R., González, J. y Pérez, S. 1994. Manual de modelos no lineales en los ámbi-tos agronómico, ganadero y forestal. Ministerio de Agricultura, Pesca y Alimenta-ción. Instituto Nacional de Investigación y Tecnología Agraria y Alimentaria. Madrid, España, p110.

Carranza, C., Lanchero, O., Miranda, D. y Chaves, B. 2009. Análisis del crecimiento de lechuga (Lactuca sativa L.) 'Batavia' cultivada en un suelo salino de la Sabana de Bogotá. Agronomía Colombiana 27 (1): 41-48. 
Carrillo, E., Mejía, J., Carballo, A., García de los Santos, G., Aguilar, V. y Corona, T. 2009. Calidad de semilla en colectas de chile de agua (Capsicum annuum L.) de los Valles centrales de Oaxaca, México. Agricultura Técnica en México 35 (3): 257-266.

Cuenca, E., Riestra, D., Pérez, J. y Echegaray, A. 1999. Crecimiento y rendimiento del tomate de cáscara (PhysalisixocarpaBrot.) regado con aguas residuales y control de la contaminación microbiana. Chapingo Serie Horticultura 5 (1): 29-34.

Delouche, J.C. 2002. Germinación, deterioro y vigor de semillas. Seed News 6(6).

Dwivedi, S., Sharma, S., Sharma, K., Singh, K. and Arun. 2012. Study on flowering and other growth behavior of brinjal (Solanummelongena) var. BR 112 in admiration to effect of C.M.L. (Country Made Liquor). J. Chem. Bio. Phy. Sci Sec. B. 2 (3): 1319-1325.

Ferreira, O., Rossi, F., Andrighetto, C. 2008. DDA-Determinador digital de áreas. Software para determinação de área foliar, índice de área foliar e área de olho de lombo. Versão 1.2. Instituto Federal de Educação, Ciéncia e tecnologia. FarroupiIha. Campus Santo Augusto. Brasil.

Gisbert, C., Prohens, J. and Nuez, F. 2011. Treatments for improving seed germination in eggplant and related species. ActaHorticulturae 898: 45-51.

Gómez, M., Baille, A., González, M. and
Mercader, J. 2003. Dry matter partitioning of greenhouse cucumber crops as affected by fruit load. ActaHorticulturae 614: 573-578.

Hartmann, H. y Kester, D. 2010. Propagación de plantas. Principios y prácticas. Compañía Editorial Continental S.A. (CECSA). México.760 p.

Hsiao, T. 1982. Spacing canopy development and light interception. Agronomy Abstracts.99 p.

Hunt, R. 1982. Plant growth analysis: second derivatives and compounded second derivatives of splined plant growth curves. Annals of Botany 50(3): 317-328.

International Seed Testing Association (ISTA). 2011. Rules proposals for the interna-tional rules for seed testing 2011 Edition. ISTA. Executive Committee. Document 05-2010-OM. Bassersdorf, Switzerland.53 p.

Kamotho, G., Muasya, M., Mathengue, W. and Dullou, E. 2003. Effects of packaging and storage conditions on seed quality of Cleome gyandra L. In: AbukutsaOnyango, M.O., Muriithi, A.N., Anjichi, V.E., Ngamau, K, Agong, S.G., Fricke, A., Hau, B. and H. Stützel (eds.). Proceedins of the Third Workshop on Sustainable Horticultural Production in the Tropics. Maseno, Kenya. p. 67-72.

Kiran, J., Vyakaranahal, B.S., Raikar, S.D., Ravikumar, G.H. and Deshpande, V.K. 2010. Seed yield and quality of brinjal as influenced by crop nutrition. 
Indian J. Agric Res. 44 (1): 1-7.

Kumar, S. 2005. Influence of pre-sowing seed treatment and seed pelleting on storability in brinjal (SolanummelongenaL.). Thesis of Master of Science (Agriculture). Dharwad India.87 p.

Martínez, N. 2006. Análisis de crecimiento y dinámica nutrimental del chile poblano (Capsicum annuum L. var. GrossumSendt) en cuatro soluciones nutritivas bajo invernadero. Tesis Maestría en Ciencias Horticultura. Universidad Autónoma Chapingo. México.

Martínez, M., Cardozo, C. y Sánchez, M. 2010. Respuesta fisiológica de semillas de tomate (Solanumlycopersicum L.) var. Unapal Maravilla y pimentón (Capsicum an-nuum L.) var. Unapal Serrano en crioconservación. ActaAgronómica 59 (4): 401-409.

Nascimento, W. 2003. Muskmelon seed germination and seedling development in response to seed priming.Scientia Agricola 60 (1): 71-75.

Nerson, H. and A. Govers. 1986. Salt priming of muskmelon seeds for low temperature germination. ScientiaHorticulturae 28(12): $85-91$.

Ngaroga, N. 2003. Effect of seed priming on germination and seedling performance of delphinium (Consolidaorientalis) cut flower.In: Abukutsa-Onyango, M.O., Muriithi, A.N., Anjichi, V.E., Ngamau, K, Agong, S.G., Fricke, A., Hau, B. and
H. Stützel (eds.). Proceedins of the third workshop on sustainable horticultural production in the tropics.Maseno, Kenya.p. 224-227.

Nieto, R. 2005. Estructura y Fisiología de las semillas. Editorial de la Universidad Autónoma Chapingo. México. 50 p.

Passam, H.C., Karavites, P.I., Papandreou, A.A., Thanos, C.A. and K. Georghiou.1989. Osmoconditioning of seeds in relation to growth and fruit yield of auber-gine, pepper, cucumber and melon in unheated greenhouse cultivation. ScientiaHorticulturae 38(3-4): 207-216.

Pérez-Camacho, I., Ayala-Garay, O., González-Hernández, V., CarrilloSalazar, J., Peña-Lomelí, A. y García de los Santos, G. 2008 a. Indicadores morfológicos y fi-siológicos del deterioro de semillas de tomate de cáscara. Agrociencia 42 (8): 891-901.

Pérez-Camacho, I., González, V., Molina, J., Ayala, O. y Peña, A. 2008 b. Efecto de desarrollo y secado de semillas de PhysalisixocarpaBrot. en germinación, vigor y contenido de azúcares. Interciencia 33 (10): 762-766.

Pichardo, J., Ayala, O., González, V., Flores, C., Carrillo, A., Peña, A., Robledo, A. y G. García.2010. Calidad fisiológica, ácidos grasos y respiración en semillas de to-mate de cáscara deterioradas artificialmente. Fitotecnia Mexicana 33 (3): 231-238.

Ponnuswamy, A.S. and Vijayalakshmi, V. 
2011. Effect of seed fortification with bioinoculants, nutrients and growth regulators on seed germination and seedling vigour of tomato (Lycopersiconesculentum), brinjal (Solanummelongena) and chilli (Capsicum annum).Madras Agricultural Journal 98 (7-9): 251-252.

Roberts, M., Long, S., Tieszen, L., and Beadle, C. 1985. Measurement of plant biomas and net primary production.pp. 1-19. In: Techniques ofbioproductivity and photosynthesis. Coombs, J., D. O. Hall, S. P. Long and J. M. O. Scurlock (eds.) Pergamonpress.

Rojas, G. y Rovalo, M. 1985. Fisiología Vegetal Aplicada. Tercera edición. McGraw-Hill. México, D.F. 302 p.

SAS Institute Inc. 2008. Statistical analysis system.The SAS@ system for Windows@ version ten.The Power to Know. Cary, NC, 27513, USA.

Sharma, S. and Sharma, K. 2010. Study on seed germination and growth behavior of brin-jalSolanummelongenavar.BR 112 in admiration to effect of C.M.L. (Country Made Liquor). Nature and Science 8 (5): 163-166.
Thuo, K. 2003. Effect of seed osmotic priming on the germination of spiderplant (Gynandropsisgynandra) seeds.In: AbukutsaOnyango, M.O., Muriithi, A.N., Anjichi, V.E., Ngamau, K, Agong, S.G., Fricke, A., Hau, B. and H. Stützel (eds.). Proceedins of the third workshop on sustainable horticultural production in the tropics. Maseno, Kenya. p. 28-32.

Torres, C., Díaz, J. y Cabal, P. 2008. Efecto de campos magnéticos en la germinación de semillas de arroz (Oryza sativa L.) y tomate (Solanumlycopersicum L.). Agronomía Colombiana 26 (2): 177-185.

Weatherbase, 2013. Montería, Colombia. En: http://www.weatherbase.com/weather/ weather.php3 $s=36008 \&$ refer $=$ \&units $=m$ etric.05-04-13.

White Harvest Seed Company, 2013. Soaking seeds for faster germination.En: http://www.whiteharvestseed.com. 0628-13.

Yeoung, Y.R., Wilson, D. and Murray, G. 1996. Germination performance and loss of late-embryogenesis-abundant (LEA) proteins during muskmelon seed priming. Seed Science and Technology 24: 429439. 